Algebraic $\& \mathcal{G}$ Geometric Topology

Volume 2 (2002) 743-755

Published: 9 September 2002

\title{
Engulfing in word-hyperbolic groups
}

\author{
G. A. NiBLO \\ B. T. Williams
}

\begin{abstract}
We examine residual properties of word-hyperbolic groups, adapting a method introduced by Darren Long to study the residual properties of Kleinian groups.
\end{abstract}

AMS Classification 20E26; 20F67, 20F65

Keywords Word hyperbolic groups, residual finiteness, engulfing

\section{Introduction}

A group is said to be residually finite if the intersection of its finite index subgroups is trivial. Equivalently it is residually finite if the trivial subgroup is closed in the profinite topology. It is an open question whether or not word hyperbolic groups are residually finite. Evidence that they may be comes from the observation that many familiar groups in this class are linear and therefore residually finite by an application of Selberg's lemma. Furthermore there are geometric methods for establishing the residual finiteness of free groups [5], surface groups [11] and some reflection groups [13] that may generalise. Nonetheless the general question seems hard to settle, hindered by the apparent difficulty of establishing that a given group contains any proper finite index subgroups at all. In [8] Long hypothesised this difficulty away by assuming that the groups he studied satisfied an engulfing property:

Definition 1.1 A subgroup $H$ in a group $G$ is said to be engulfed if $H$ is contained in a proper finite index subgroup of $G$. The group $G$ has the engulfing property with respect to a class $\mathcal{H}$ of subgroups of $G$ if every subgroup in the class $\mathcal{H}$ is engulfed in $G$.

As we will later see Long was able to deduce a strengthened form of residual finiteness for certain Kleinian groups satisfying a relatively mild engulfing hypothesis. In [7] Kapovich and Wise showed that the question of residual finiteness for the class of word hyperbolic groups could be reduced to a question concerning engulfing. 
Theorem (Kapovich, Wise) The following are equivalent:

(i) Every word-hyperbolic group is residually finite.

(ii) Every word-hyperbolic group has at least one proper finite index subgroup.

The second condition is equivalent to the assertion that every word hyperbolic group engulfs the identity. While the result of Kapovich and Wise offers the possibility of an attack on the question of residual finiteness for the class of word-hyperbolic groups, there is a real possibility that non-residually finite word hyperbolic groups exist. In this paper we show how to tackle the more restricted question of whether a given word-hyperbolic group is residually finite by suitably adapting Long's method to obtain the following:

Theorem 4.1 Let $G$ be a word-hyperbolic group and suppose that $G$ engulfs every finitely generated free subgroup with limit set a proper subset of the boundary of $G$. Then the intersection of all finite index subgroups of $G$ is finite. If $G$ is torsion free then it is residually finite.

It is hoped that this result may lead to a new attack on the question of residual finiteness for certain classes of word hyperbolic groups.

Long's principal aim in introducing engulfing was to establish much stronger residual properties. A subgroup $H$ of a group $G$ is said to be separable in $G$ if it is an interesection of finite index subgroups (equivalently $H$ is closed in the profinite topology on $G$ ). Residual finiteness is equivalent to separability of the trivial subgroup.

Theorem (Long) Let $\Gamma$ be the fundamental group of a closed hyperbolic 3manifold. Suppose that $\Gamma$ has the engulfing property for those finitely generated subgroups $H$ with $\Lambda(H)<S_{\infty}^{2}$. Then any geometrically finite subgroup of $\Gamma$ has finite index in its profinite closure.

There has been substantial recent progress in the field:

- In [4] Gitik showed how to construct examples of closed hyperbolic 3manifolds such that every quasi-convex subgroup of the fundamental group is closed in the profinite topology. Gitik builds the manifolds by a sequence of doubling operations each of which consists of glueing two copies of a given compact hyperbolic 3-manifold with non-empty boundary along an incompressible subsurface of the boundary. Gitik showed 
that, given appropriate constraints on the glueing, the fundamental group of the doubled manifold has the property that all of its quasi-convex subgroups are closed in the profinite topology. Starting with a handlebody (the fundamental group of which is free and therefore subgroup separable by Hall's theorem, [5]), Gitik constructs sequences of doubling operations which yield examples of closed hyperbolic 3-manifolds with fundamental groups satisfying this property.

- In [15] Wise showed that every quasi-convex subgroup of the fundamental group of the Figure 8 knot complement is closed in the profinite topology using a geometric method which generalises to many other link complements, and indeed to other examples arising in geometric group theory. The conclusion is subsumed by the result of Long and Reid [10].

- Using arithmetic techniques and building on a method suggested by the paper of Scott [13], Agol, Long and Reid [1] showed that the geometrically finite subgroups of Bianchi groups are closed in the profinite topology.

In our second main result we again adapt Long's technique to show:

Theorem 5.2 Let $G$ be a word-hyperbolic group which engulfs every finitely generated subgroup $K$ such that the limit set $\Lambda(K)$ is a proper subset of the boundary of $G$. Then every quasi-convex subgroup of $H$ has finite index in its profinite closure in $G$.

It may be that existing proofs of separability can be simplified using this result, but by way of caution we also generalise a construction of Long's to show that every non-elementary word hyperbolic group contains proper subgroups which fail to be engulfed. However the construction sheds no light on the question of engulfing for finitely generated subgroups.

The work of adapting Long's argument to the context of torsion free word hyperbolic groups formed part of the thesis of the second author [14]. The main technical difficulties in this paper arise in adapting the argument to the presence of torsion.

\section{Word-hyperbolic groups}

This section is a brief introduction to word-hyperbolic groups. The reader is referred to [3] for a full treatment. 
Let $G$ be a finitely generated group, let $S$ be a finite generating set for $G$, and consider $G$ as a metric space with respect to the word metric corresponding to this generating set.

The group $G$ is said to be word-hyperbolic if it is a $\delta$-hyperbolic space for some $\delta \geq 0$.

The boundary at infinity of $G$, denoted $\partial G$ is defined as a metric space whose points are equivalence classes of rays converging to infinity in the group. It is the dynamics of the action of $G$ (and its subgroups) on this boundary that we will use to prove the main theorems in this paper. We take a moment to recall the important features of the boundary and of those dynamics.

A word-hyperbolic group is called elementary if it is finite or contains a finite index infinite cyclic subgroup and is non-elementary otherwise. An elementary word-hyperbolic group is either finite, in which case it has an empty boundary at infinity, or it is virtually cyclic in which case its boundary consists of two points. For any word hyperbolic group the boundary is compact and metrisable, and non-elementary word-hyperbolic groups have infinite boundaries in which there are no isolated points.

Given a subgroup $H$ of $G$, the limit set of $H$ which is denoted $\Lambda(H)$ is defined as the subset of $\partial G$ attainable by sequences of elements of $H$. $H$ acts properly discontinuously on $\partial G \backslash \Lambda(H)$.

The following describes the action of infinite order elements on the boundary. If $g$ is an infinite order element of $G$ it acts on the Cayley graph $G$ by translation along a quasi-geodesic line, $\alpha$ say, (obtained by joining $g^{i}$ to $g^{i+i}$ for all $i \in \mathbb{Z}$ by a geodesic in $G)$. Denote by $\partial g=\left\{\partial g^{+}, \partial g^{-}\right\}=\left\{\lim _{i \rightarrow \infty} g^{i}, \lim _{i \rightarrow \infty} g^{-i}\right\}$ the endpoints of $\alpha$ in $\partial G$ (which are fixed by $g$ ). There exist disjoint neighbourhoods $U_{+}$and $U_{-}$of $\partial g^{+}$and $\partial g^{-}$respectively such that for sufficiently large $r$ and all $x \in \partial G \backslash\left(U_{+} \cup U_{-}\right)$we have $g^{r} x \in U_{+}$and $g^{-r} x \in U_{-}$. We say that the pair $\left(U_{+}, U_{-}\right)$is absorbing for $g^{r}$. In fact any pair of disjoint neighbourhoods of $\partial g^{+}$and $\partial g^{-}$is absorbing for $g^{k}$ for sufficiently large $k$. (See [3] Chapter 8.)

The following well known fact can be viewed as an alternative definition of the limit set of a subgroup. A proof is included for the convenience of the reader.

Lemma 2.1 Let $H$ be a non-elementary subgroup of a word-hyperbolic group $G$. Then $\Lambda(H)$ is the smallest non-empty closed $H$-invariant subset of $\partial G$.

Proof We prove that if $A \subseteq \partial G$ is closed and $H$-invariant then $\Lambda(H) \subseteq A$. Firstly, let $B \subset \partial G$. Denote by $I(B)$ the set of points of $G$ lying on geodesics 
between points of $B$. Suppose that $B \neq \emptyset$ and $|B| \neq 1$. Then $I(B) \neq \emptyset$. Let $\left\{x_{i}\right\} \subseteq I(B)$ be a sequence such that $x_{i} \rightarrow x \in \partial G$. We claim that $x \in \bar{B}$. To see this, for each $i$ choose a geodesic $l_{i}=\left[b_{i}^{\prime}, b_{i}^{\prime \prime}\right]$ with $b_{i}^{\prime}, b_{i}^{\prime \prime} \in B$. Passing to a subsequence if necessary we get $b_{i}^{\prime} \rightarrow b^{\prime} \in \bar{B}, b_{i}^{\prime \prime} \rightarrow b^{\prime \prime} \in \bar{B}, l_{i} \rightarrow l$. $x_{i} \rightarrow x \in l \cup\left\{b^{\prime}, b^{\prime \prime}\right\}$ and hence $x \in\left\{b^{\prime}, b^{\prime \prime}\right\}$.

Now let $A \subseteq \partial G$ be closed and $H$-invariant. Let $I(A)$ be as above. Then $I(A)$ is $H$-invariant. First suppose that $1 \in I(A)$. Then $H \subseteq I(A)$. Let $x \in \Lambda(H)$ and $\left\{x_{i}\right\} \subseteq H$ so that $x_{i} \rightarrow x$. By the first paragraph of the proof $x \in \bar{A}=A$ and hence $\Lambda(H) \subseteq A$. Now suppose that $1 \notin I(A)$. Then $I(A) \cap H=\emptyset$ and $I(A)$ is a union of right cosets of $H$. Suppose that $H g \subseteq I(A)$. Let $x \in \Lambda(H)$ and $\left\{x_{i}\right\} \subseteq H$ with $x_{i} \rightarrow x$. Then since $x_{i} g$ and $x_{i}$ are a distance exactly $|g|$ apart for all $i$ we have $x_{i} g \rightarrow x \in \Lambda(H)$ and hence $x \in \bar{A}=A$ and $\Lambda(H) \subseteq A$ as required.

It is clear that $\Lambda(H)$ is $H$-invariant so it remains to prove that $\Lambda(H)$ is closed. We show that $\partial G \backslash \Lambda(H)$ is open. Let $y \in \partial G \backslash \Lambda(H)$ and let $\left\{y_{i}\right\}$ be a sequence converging to $y$. Let $\alpha_{i}$ be geodesics realising the distances $d\left(y_{i}, H\right)$. There is no bound on the lengths of the $\alpha_{i}$. Let $z_{i}$ lie on $\alpha_{i}$ so that there is no bound on the distances $d\left(y_{i}, z_{i}\right)$ and $d\left(z_{i}, H\right)$. Let $\left\{z_{i}\right\}$ converge to $z \in \partial G$ then the horoball $N_{(y . z)}(y)$ is an open set containing $y$ and disjoint from $\Lambda(H)$ as required.

Corollary 1 Let $H$ be a non-elementary subgroup of a word-hyperbolic group $G$. Then $\Lambda(H)$ is the closure of the set

$$
S=\left\{\partial h^{+}, \partial h^{-} \mid h \in H, h \text { has infinite order }\right\} \subset \partial G .
$$

Proof By Lemma $2.1 \Lambda(H)$ is the minimum non-empty closed $H$-invariant subset of $\partial G$. For any infinite order element $h \in H$, the limit points $\partial h^{ \pm}$both lie in $\Lambda H$, hence the closure of $S$ must be contained in $\Lambda(H)$. On the other hand $S$ is clearly $H$-invariant and so by Lemma 2.1 its closure contains $\Lambda(H)$ as required.

We will need the following technical observation:

Lemma 2.2 Let $G$ be a non-elementary word-hyperbolic group with generators $g_{1}, \ldots g_{n}$, and $N$ a subgroup of $G$ with $\Lambda N=\partial G$. Then there are infinite order elements $x_{1}, \ldots x_{n}$ in $N$ such that the elements $x_{i} g_{i} x_{i}$ generate a free subgroup $H<G$ with $\Lambda H \neq \partial G$. In particular $G$ is generated by the subset $\left\{x_{1}, \ldots x_{n}, x_{1} g_{1} x_{1}, \ldots x_{n} g_{n} x_{n}\right\}$ which consists of elements of infinite order. 
Proof Since $G$ is non-elementary its boundary is infinite, and since limit sets of infinite order elements of $N$ are dense, given any non-empty open subset $\mathcal{U}$ of the boundary we may choose elements $y_{1}, \ldots, y_{n} \in N$ of infinite order with $\partial y_{i} \subseteq \mathcal{U}$ for all $i$ and $\partial y_{i} \cap \partial y_{j}=\emptyset$ for $i \neq j$. In particular if we let $\mathcal{U}$ be the complement in $\partial G$ of the union of the fixed sets of the infinite order elements in the set $\left\{g_{1}, \ldots, g_{n}\right\}$ then we can also ensure that $\partial g_{i} \cap \partial y_{j}=\emptyset$ for all $i, j$

If a generator $g_{i}$ acts trivially on the boundary then set $x_{i}=y_{i}$. The element $x_{i} g_{i} x_{i}$ acts on the boundary in the same way as the infinite order element $y_{i}^{2}$, and its two fixed points are $\partial y_{i}^{ \pm} \in \mathcal{U}$. If the generator $g_{i}$ has infinite order then since its fixed points are disjoint from those of $y_{i}$ (and the boundary is metrisable), we may choose small neighbourhoods $\mathcal{U}_{i}^{ \pm}$of the limit points $\partial y_{i}^{ \pm}$ such that $g_{i}^{ \pm 1}\left(\mathcal{U}_{i}^{+} \cup \mathcal{U}_{i}^{-}\right) \cap\left(\mathcal{U}_{i}^{+} \cup \mathcal{U}_{i}^{-}\right)=\emptyset$. We choose the neighbourhoods $\mathcal{U}_{i}^{ \pm}$ small enough to be disjoint and so that the complement of the closure of the union of the neighbourhoods is non-empty.

The neighbourhoods $\mathcal{U}_{i}^{ \pm}$are absorbing for any sufficiently high power $y_{i}^{ \pm r}$ of $y_{i}$, and it follows easily that setting $x_{i}=y_{i}^{r}$ the neighbourhoods are absorbing for $\left(x_{i} g_{i} x_{i}\right)^{ \pm 1}$. To see this choose any point $p$ in the complement of $\mathcal{U}_{i}^{+} \cup \mathcal{U}_{i}^{-}$. Its image $x_{i}(p)$ lies in $\mathcal{U}_{i}^{+}$, and since $g_{i}\left(\mathcal{U}_{i}^{+} \cup \mathcal{U}_{i}^{-}\right) \cap\left(\mathcal{U}_{i}^{+} \cup \mathcal{U}_{i}^{-}\right)=\emptyset g_{i} x_{i}(p)$ does not lie in $\mathcal{U}_{i}^{+} \cup \mathcal{U}_{i}^{-}$. Hence $x_{i} g_{i} x_{i}(p)$ lies in $\mathcal{U}_{i}^{+}$. A similar argument shows that $x_{i}^{-1} g_{i}^{-1} x_{i}^{-1}(p)$ lies in $\mathcal{U}_{i}^{-}$, and iterating shows that $\left(x_{i} g_{i} x_{i}\right)^{r}(p) \in \mathcal{U}_{i}^{+} \cup \mathcal{U}_{i}^{-}$for any non-zero power of the element $x_{i} g_{i} x_{i}$.

We will now use the standard Schottky argument to show that these elements generate a free subgroup. Let $w=x_{i_{1}}^{\epsilon_{i_{1}}} g_{i_{1}}^{\epsilon_{i_{1}}} x_{i_{1}}^{\epsilon_{i_{1}}} \ldots x_{i_{s}}^{\epsilon_{i_{s}}} g_{i_{s}}^{\epsilon_{i_{s}}} x_{i_{s}}^{\epsilon_{i_{s}}}$ be a reduced word in the elements $x_{i} g_{i} x_{i}$ and their inverses, and choose a point $p$ in the complement of the union of the absorbing pairs $\mathcal{U}_{i}^{+} \cup \mathcal{U}_{i}^{-}$. As argued above $x_{i_{s}}^{\epsilon_{i_{s}}} g_{i_{s}}^{\epsilon_{i_{s}}} x_{i_{s}}^{\epsilon_{i_{s}}}(p) \in \mathcal{U}_{i_{s}}^{+} \cup \mathcal{U}_{i_{s}}^{-}$. If $i_{s-1}=i_{s}$ then we may iterate to see that the image of $p$ under the element $x_{i_{s-1}}^{\epsilon_{i_{s-1}}} g_{i_{s-1}}^{\epsilon_{i_{s-1}}} x_{i_{s-1}}^{\epsilon_{i_{s-1}}}$ also lies in $\mathcal{U}_{i_{s}}^{+}=\mathcal{U}_{i_{s-1}}^{+}$. If $i_{s-1} \neq i_{s}$ then, since the absorbing set $\mathcal{U}_{i_{s}}^{+} \cup \mathcal{U}_{i_{s}}^{-}$is disjoint from the absorbing set $\mathcal{U}_{i_{s-1}}^{+} \cup \mathcal{U}_{i_{s-1}}^{-}$, the image $x_{i_{s-1}}^{\epsilon_{i_{s-1}}} g_{i_{s-1}}^{\epsilon_{i_{s-1}}} x_{i_{s-1}}^{\epsilon_{i_{s-1}}} \ldots x_{i_{s}}^{\epsilon_{i_{s}}} g_{i_{s}}^{\epsilon_{i_{s}}} x_{i_{s}}^{\epsilon_{i_{s}}}(p)$ lies in $\mathcal{U}_{i_{s-1}}^{+} \cup \mathcal{U}_{i_{s-1}}^{-}$. Iterating the argument we see that the point $p$ ends in the absorbing pair $\mathcal{U}_{i_{1}}^{+} \cup \mathcal{U}_{i_{1}}^{-}$. Since it did not start there it is not invariant under the action of the element $w$ which is therefore not the identity. Hence every reduced word in the generators $x_{i} g_{i} x_{i}$ is non-trivial and the subgroup is free as required. Finally we note that since the accumulation points for the action of this subgroup $H$ lie in the union of the absorbing pairs $\mathcal{U}_{i}^{+} \cup \mathcal{U}_{i}^{-}$the limit set of this subgroup lies in the closure of their union. Since this closure is not all of $\partial G$ neither is $\Lambda H$. 


\section{Separability}

The profinite topology on a group $G$ is defined by taking as a basis for the closed sets the cosets of all finite index normal subgroups of $G$. Note that finite index subgroups are themselves closed, and (since the complement is a finite union of cosets each of which is also open) they are also open. Given a subgroup $H<G$ we will denote the closure of $H$ in the profinite topology on $G$ by $\bar{H}$.

Definition 3.1 Given a group $G$, a finitely generated subgroup $H$ is separable in $G$ if it is closed in the profinite topology on $G$. A group $G$ is residually finite if $\{e\}$ is closed and is subgroup separable or LERF (locally extended residually finite) if every finitely generated subgroup $H$ is separable in $G$. A wordhyperbolic group is qc subgroup separable if every quasi convex subgroup is closed in the profinite topology.

Note that if a group is subgroup separable then a fortiori it has the engulfing property for its finitely generated subgroups. On the other hand in [12] examples are given of fundamental groups of geometric 3-manifolds which contain two generator subgroups which are not even engulfed. These examples, based on earlier examples of [2] are not word-hyperbolic, however Long showed in [8] that the fundamental group of a hyperbolic 3-manifold always contains (infinitely generated) subgroups that are not engulfed.

\section{4 (Almost) residual finiteness}

For this section let $N$ denote the residual core of $G$, i.e., intersection of all finite index subgroups. (This is the closure $\overline{\{e\}}$ of the trivial subgroup in the profinite topology.) This subgroup is normal and therefore [3] its limit set is either empty (if $N$ is finite) or all of $\partial G$ (if $N$ is infinite).We will say that the group $G$ is almost residually finite if $N$ is a finite subgroup. Note that torsion free almost residually finite groups are residually finite.

Theorem 4.1 Let $G$ be a word-hyperbolic group and suppose that $G$ engulfs every finitely generated free subgroup $S$ such that $\Lambda(S)$ is a proper subset of $\partial G$. Then $G$ is almost residually finite. If $G$ is torsion free, then it is residually finite. 
Proof If $G$ is elementary then it is either finite or virtually cyclic. In both cases it is trivially residually finite, so we may assume that $G$ is non-elementary and has infinite boundary.

Let $\left\{g_{i} \mid 1 \leq i \leq n\right\}$ be a generating set for $G$. If $G$ is not almost residually finite then $\Lambda(N)=\partial G$. It follows from Lemma 2.2 that we may choose elements $x_{i} \in N$ such that the elements $x_{i} g_{i} x_{i}$ generate a free subgroup $H$ with $\Lambda H \neq$ $\partial G$. By hypothesis $H$ is engulfed, so there is a proper finite index subgroup $L<G$ with $H<L$. The subgroup $L$ must contain the elements $x_{i} g_{i} x_{i}$, but by hypothesis $N<L$ so it also contains the elements $x_{i}$. Hence it contains all of the generators $g_{i}$ of $G$. This is a contradiction. Hence $G$ is almost residually finite, and if $G$ is torsion free it is residually finite.

\section{5 (Almost) subgroup separability}

Note that if $H$ is a finite subgroup of an almost residually finite group $G$, and if $N$ is the intersection of the finite index subgroups of $G$, then $H N$ is finite, and is closed. Hence the intersection of the finite index subgroups of $G$ containing $H$ is a finite extension of $H$.

Definition 5.1 We will say that a subgroup $H<G$ is almost separable if $H$ has finite index in $\bar{H}$.

Theorem 5.2 Let $G$ be a non-elementary word-hyperbolic group. Suppose that $G$ has the engulfing property for all finitely generated subgroups $K$ such that $\Lambda(K)$ is a proper subset of $\partial G$. Then every quasi-convex subgroup $H \leq G$ is almost separable in $G$.

Proof Applying Theorem 4.1 we see that the intersection $N$ of all finite index subgroups of $G$ is finite. It is easy to see that $G / N$ is itself residually finite.

Let $K N / N$ be any subgroup of $G / N$ with limit set a proper subset of the boundary of $G / N$. There is a G-equivariant quasi-isometry from $G$ to $G / N$ taking $K N$ to $K N / N$ and it follows that the limit set of $K N$ is a proper subset of the boundary of $G$. By the hypothesis there is a proper finite index subgroup of $G$ containing $K N$, and since it contains $N$ its image is a proper finite index subgroup of $G / N$ containing $K N / N$. Hence $G / N$ satisifes the hypotheses of the theorem, but in addition it is residually finite.

Now suppose the theorem is true for $G / N$. Let $H$ be a quasi-convex subgroup of $G$, so $H N / N$ is a quasi-convex subgroup of $G / N$. By the assumption, $H N / N$ 
has finite index in its closure $\overline{H N / N}$ under the profinite topology. Since the map $G \longrightarrow G / N$ is continuous the preimage of $\overline{H N / N}$ is itself closed in $G$ and clearly contains $H$ as a subgroup of finite index. Hence in order to establish the theorem for $G$ it suffices to establish it for $G / N$. This reduces us to the case where $G$ is residually finite, so from now on we make this additional assumption.

Now since $G$ is residually finite, its finite subgroups and its maximal abelian subgroups (see [9]) are all closed in the profinite topology. Since $G$ is wordhyperbolic its maximal abelian subgroups are virtually cyclic, and therefore every elementary subgroup of $G$ has finite index in its profinite closure. Hence we can assume that $H$ is non-elementary.

We will make use of the following observation. A proof is given in Kapovich and Short [6].

Lemma 5.3 Let $H$ be a quasiconvex subgroup of a word-hyperbolic group $G$. If $H<L<G$ with $\Lambda(H)=\Lambda(L)$ then $|H: L|<\infty$.

It follows from this that it suffices to show that the profinite closure $\bar{H}$ of any non-elementary quasi-convex subgroup $H<G$ has the same limit set as $H$. For the remainder of the argument fix a generating set $\left\{g_{1}, g_{2}, \ldots, g_{n}\right\}$ for $G$. By Lemma 2.2 we can choose this set to consist of infinite order elements.

Since $H \leq \bar{H}$ clearly $\Lambda(H) \subseteq \Lambda(\bar{H})$, and if $\Lambda(H)=\partial G$ then the result is clear so suppose that $\Lambda(H)$ is a proper subset of $\partial G$. Assume, for a contradiction, that $\Lambda(H) \neq \Lambda(\bar{H})$.

Choose a point $p \in \Lambda(\bar{H}) \backslash \Lambda(H)$. By Corollary 1 there is a sequence of infinite order elements $k_{i} \in \bar{H}$ with fixed points $p_{i} \in \Lambda(\bar{H}) \subset \partial G$ such that the sequence $p_{i}$ converges to $p$. Since $\Lambda(H)$ is closed and $p \notin \Lambda H$ almost all the points $p_{i}$ are also not in $\Lambda(H)$, so almost all the elements $k_{i}$ are in $\bar{H} \backslash H$ and, since limit sets of non-elementary quasi-convex subgroups have no isolated limit points, without loss we can choose them to have distinct limit sets. Hence we can choose one of them with limit points $p^{ \pm}$in $\partial G$ distinct from the limit points of the generators. Since $p^{ \pm}$are also not in $\Lambda(H)$ we may choose an absorbing pair of neighbourhoods $U^{ \pm}$of the pair $p^{ \pm}$disjoint from $\Lambda(H) \cup\left\{g_{1}, g_{2}, \ldots, g_{n}\right\}$. Since $G$ acts uniformly on its boundary and $\Lambda H$ is a closed set disjoint from the limit points of $k$, for some power $k^{r}$ the image $k^{r}(\Lambda(H))$ is contained in $U^{+}$and is therefore disjoint from $\Lambda(H) \cup\left\{g_{1}, g_{2}, \ldots, g_{n}\right\}$. The image $k^{r}(\Lambda(H))$ is the limit set of $k^{r} H k^{-r}$ which by construction is a subgroup of $\bar{H}$.

Since $H$ is non-elementary so is $k^{r} H k^{-r}$ and we may choose elements $y_{1}, y_{2}$, $\ldots, y_{n} \in k^{r} H k^{-r}$ with distinct fixed sets in the boundary. Notice that by our 
construction of the subgroup $k^{r} H k^{-r}$ the fixed points $\partial y_{1}, \partial y_{2}, \ldots, \partial y_{n}$ lie in $\Lambda(\bar{H})-\Lambda(H)$ and $\partial y_{i} \neq \partial g_{j}$ for any $i, j$. We may later need to modify the choice of these elements by taking powers of them. In doing so we do not change their fixed points.

Let $C \subset \partial G-\Lambda(H)$ be a compact set containing the fixed points of the elements $y_{i}$ in its interior (the closure of a sufficiently small open metric ball around the fixed points will do). $H$ acts properly discontinuously on $\partial G-\Lambda(H)$ so there are finitely many non-trivial elements of $H, h_{1}, h_{2}, \ldots h_{m}$ say, taking $C$ to intersect itself. Since $G$ is residually finite so is $H$, and so there exists a finite index normal subgroup $A \triangleleft H$ containing none of the $h_{i}$.

We now need the following technical Lemma taken from [8].

Lemma 5.4 Let $G$ and $H$ be as above and suppose that $A \triangleleft H$ is a normal subgroup of index $t$ in $H$. For any element $h \in \bar{H}, h^{t} \in \bar{A}$.

Since taking powers of the elements $y_{i}$ does not change their fixed points we can use this lemma to ensure that the elements $y_{i}$ all lie in the subgroup $\bar{A}$. Since $\partial G$ is metrisable we can choose $n$ mutually disjoint pairs of neighbourhoods $\left(U_{i}^{+}, U_{i}^{-}\right)$for the $\partial y_{i}$ so that the closure of each is contained in the interior of $C$. Ensure that $\left(U_{i}^{+}, U_{i}^{-}\right)$is absorbing for $y_{i}$ by again taking large powers and relabelling.

Now let $s_{i}=y_{i} g_{i} y_{i}$ and consider the group $B$ generated by the elements $s_{i}$ together with the generators of $A$. Since $A$ has finite index in the finitely generated group $H$ it too is finitely generated and so is $B$. We claim that its limit set is contained in the closure of $\cup_{i}\left(U_{i}^{+}, U_{i}^{-}\right) \cup(\partial G-C)$.

Let $U_{i}=U_{i}^{+} \cup U_{i}^{-}$.

The limit set is the closure of the H-orbit of any point in it (by 1). Choose a point $p \in C-\cup_{i} U_{i}$ and write an arbitrary element $b \in B$ as a reduced word $s_{i_{1}}^{\epsilon_{1}} a_{1} s_{i_{2}}^{\epsilon_{2}} a_{2} \ldots s_{i_{k}}^{\epsilon_{k}} a_{k}$ where $a_{i} \in A$, where possibly $s_{i_{1}}$ or $a_{k}$ may be the identity elements, but none of the other elements are trivial. We examine the image of $p$ under the action of $b$; there are four cases to consider:

Neither $s_{i_{1}}$ nor $a_{k}$ is the identity:

$$
\begin{aligned}
b(p) & =s_{i_{1}}^{\epsilon_{1}} a_{1} s_{i_{2}}^{\epsilon_{2}} a_{2} \ldots s_{i_{k}}^{\epsilon_{k}} a_{k}(p) \in s_{i_{1}}^{\epsilon_{1}} a_{1} s_{i_{2}}^{\epsilon_{2}} a_{2} \ldots s_{i_{k}}^{\epsilon_{k}}(\partial G-C) \\
& \subset s_{i_{1}}^{\epsilon_{1}} a_{1} s_{i_{2}}^{\epsilon_{2}} a_{2} \ldots s_{i_{k}}^{\epsilon_{k}}\left(\partial G-\left(U_{i_{k}}\right)\right) \subset s_{i_{1}}^{\epsilon_{1}} a_{1} s_{i_{2}}^{\epsilon_{2}} a_{2} \ldots a_{i_{k-1}} U_{i_{k}} \\
& \subset s_{i_{1}}^{\epsilon_{1}} a_{1} s_{i_{2}}^{\epsilon_{2}} a_{2} \ldots a_{i_{k-1}} C \subset s_{i_{1}}^{\epsilon_{1}} a_{1} s_{i_{2}}^{\epsilon_{2}} a_{2} \ldots s_{i_{k-1}}(\partial G-C) \\
& \vdots \\
& \subset s_{i_{1}}^{\epsilon_{1}} a_{1} C \subset s_{i_{1}}^{\epsilon_{1}}(\partial G-C) \subset s_{i_{1}}^{\epsilon_{1}}\left(\partial G-\left(U_{i_{1}}\right)\right) \subset U_{i_{1}}
\end{aligned}
$$


Only $a_{k}$ is the identity:

$$
\begin{aligned}
& b(p)=s_{i_{1}}^{\epsilon_{1}} a_{1} s_{i_{2}}^{\epsilon_{2}} a_{2} \ldots s_{i_{k}}^{\epsilon_{k}} a_{k}(p) \in s_{i_{1}}^{\epsilon_{1}} a_{1} s_{i_{2}}^{\epsilon_{2}} a_{2} \ldots s_{i_{k}}^{\epsilon_{k}}\left(C-\cup_{i} U_{i}\right) \\
& \quad \subset s_{i_{1}}^{\epsilon_{1}} a_{1} s_{i_{2}}^{\epsilon_{2}} a_{2} \ldots a_{i_{k-1}} U_{i_{k}} \subset s_{i_{1}}^{\epsilon_{1}} a_{1} s_{i_{2}}^{\epsilon_{2}} a_{2} \ldots a_{i_{k-1}} C \\
& \quad \subset s_{i_{1}}^{\epsilon_{1}} a_{1} s_{i_{2}}^{\epsilon_{2}} a_{2} \ldots s_{i_{k-1}}^{\epsilon_{k-1}}(\partial G-C) \\
& \quad \vdots \\
& \quad \subset s_{i_{1}}^{\epsilon_{1}} a_{1} C \subset s_{i_{1}}^{\epsilon_{1}}(\partial G-C) \subset s_{i_{1}}^{\epsilon_{1}}\left(\partial G-\left(U_{i_{1}}\right)\right) \subset U_{i_{1}}
\end{aligned}
$$

Only $s_{i_{1}}$ is the identity:

$$
\begin{aligned}
& b(p)=a_{1} s_{i_{2}}^{\epsilon_{2}} a_{2} \ldots s_{i_{k}}^{\epsilon_{k}} a_{k}(p) \in a_{1} s_{i_{2}}^{\epsilon_{2}} a_{2} \ldots s_{i_{k}}^{\epsilon_{k}}\left(C-\cup_{i} U_{i}\right) \subset a_{1} s_{i_{2}}^{\epsilon_{2}} a_{2} \ldots a_{i_{k-1}} U_{i_{k}} \\
& \quad \subset a_{1} s_{i_{2}}^{\epsilon_{2}} a_{2} \ldots a_{i_{k-1}} C \subset a_{1} s_{i_{2}}^{\epsilon_{2}} a_{2} \ldots s_{i_{k-1}}(\partial G-C) \\
& \quad \quad \vdots \\
& \quad \subset a_{1} C \subset(\partial G-C)
\end{aligned}
$$

Both $s_{i_{1}}$ and $a_{k}$ are the identity:

$$
\begin{aligned}
b(p) & =a_{1} s_{i_{2}}^{\epsilon_{2}} a_{2} \ldots s_{i_{k}}^{\epsilon_{k}} a_{k}(p) \in a_{1} s_{i_{2}}^{\epsilon_{2}} a_{2} \ldots s_{i_{k}}^{\epsilon_{k}}\left(C-\cup_{i} U_{i}\right) \subset a_{1} s_{i_{2}}^{\epsilon_{2}} a_{2} \ldots a_{i_{k-1}} U_{i_{k}} \\
& \subset a_{1} s_{i_{2}}^{\epsilon_{2}} a_{2} \ldots a_{i_{k-1}} C \subset a_{1} s_{i_{2}}^{\epsilon_{2}} a_{2} \ldots s_{i_{k-1}}(\partial G-C) \\
\quad & \quad \therefore \\
& \subset a_{1} C \subset(\partial G-C)
\end{aligned}
$$

The conclusion is that $p$ ends up in $\cup_{i} U_{i}$ or in $\partial G-C$, and in particular the closure of its orbit lies in the union of the closures of these subsets as required.

Hence $B$ is a finitely generated subgroup of $G$ with $\Lambda(B)$ a proper subset of $\partial G$ and our engulfing hypothesis for such subgroups ensures that there exists a proper finite index subgroup $K<G$ containing $B$. Since this subgroup contains $A$ it also contains $\bar{A} \leq K$ and hence $K$ contains the elements $y_{1}, y_{2}, \ldots, y_{n}$. But $K$ also contains the elements $s_{i}=y_{i} g_{i} y_{i}$ and hence contains all of the generators of $G$. So $K=G$ contradicting the fact that $K$ is a proper subgroup.

\section{A non-engulfed proper (locally-free) subgroup}

In this section we show that every non-elementary word hyperbolic group contains subgroups which are not engulfed. More generally we show:

Theorem 6.1 Let $G$ be a non-elementary word hyperbolic group and $\mathcal{F}$ a countable collection of quotients of $G$ each with infinite kernel. Then $G$ contains a proper (infinitely generated) subgroup $K$ which surjects on every quotient in the family $\mathcal{F}$. In particular $G$ contains a proper subgroup $K$ which is not engulfed. 
Proof Enumerate the kernels of the quotients, and for each kernel choose a set of left coset representatives. Since $G$ is finitely generated each such set is countable, and we can enumerate the union of the sets of coset representatives as $g_{i}, i \in \mathbb{N}$ with associated kernels $N_{i}$.

Choose a proper open subset $\mathcal{U}$ in $\partial G$. Since the kernels are all infinite the limit set of each kernel is dense in the boundary of $G$. Hence given any finite subset $S_{i} \subset \mathcal{U}$ we can choose an infinite order element $y_{i} \in N_{i}$ such that $\partial y_{i} \subset \mathcal{U} \backslash S$ and $\partial y_{i} \cap \partial g_{i}=\emptyset$. Now for sufficiently high powers $y_{i}^{r_{i}}$ of $y_{i}$ and any point $p \notin \partial y_{i}$ the image $y_{i}^{r_{i}} g_{i} y_{i}^{r_{i}}(p)$ lies in $\mathcal{U}$, hence the limit set of all these elements lies in $\mathcal{U}$. Setting the subset $S_{i}=\bigcup_{j=1}^{i} \partial y_{j}$ we may choose these elements $y_{i}$ and their powers $r_{i}$ inductively to ensure that the subset $\left\{y_{i}^{r_{i}} g_{i} y_{i}^{r_{i}} \mid i=1, \ldots n\right\}$ freely generates a subgroup of $G$ with limit set contained in $\mathcal{U}$, just as we did in Lemma 2.2. (Again care must be taken over the choice of absorbing pairs for the elements and we may need to raise the power of the elements $y_{i}$.)

It follows that the subgroup generated by any finite subset of these elements has limit set contained in $\mathcal{U}$. Any element of the subgroup $K$ generated by all of these elements lies in one of these finitely generated subgroups and therefore has its limit set insde $\mathcal{U}$. Applying Corollary 1 we see that $\Lambda K$ is a proper subset of $\partial G$ and so $K$ is a proper (indeed infinite index) subgroup of $G$.

Consider the image of this subgroup in one of the quotients $G / N \in \mathcal{F}$. By construction for each left coset representative $g$ of the subgroup $N$, the subgroup $K$ contains a generator $y^{r} g y^{r}$ for some element $y \in N$ so $K$ contains a full set of left coset representatives for each of the kernels in $\mathcal{F}$ as required.

Now setting $\mathcal{F}$ to be the set of finite quotients of $G$ we obtain a proper subgroup which surjects on every finite quotient, and hence is not engulfed. The ping-pong construction applied at each stage of the argument shows that we can ensure that the subgroup is an ascending union of finitely generated free subgroups, and is therefore locally free.

Note that the subgroup $K$ constructed in the theorem cannot be finitely generated since if it were then the ascending chain of subgroups generated by the finite subsets $\left\{y_{i}^{r_{i}} g_{i} y_{i}^{r_{i}} \mid i=1, \ldots n\right\}$ would terminate, which it does not do by construction. 


\section{References}

[1] I Agol, D D Long, A W Reid, The Bianchi groups are separable on geometrically finite subgroups, Ann. of Math. (2) 153 (2001) 599-621

[2] R G Burns, A Karrass, D Solitar, A note on groups with separable finitely generated subgroups, Bull. Austral. Math. Soc. 36 (1987) 153-160

[3] É Ghys, P de la Harpe (editors), Sur les groupes hyperboliques d'après Mikhael Gromov, Birkhäuser Boston Inc., Boston, MA (1990), papers from the Swiss Seminar on Hyperbolic Groups held in Bern, 1988

[4] Rita Gitik, Doubles of groups and hyperbolic LERF 3-manifolds, Ann. of Math. (2) 150 (1999) 775-806

[5] Marshall Hall, Jr, Coset representations in free groups, Trans. Amer. Math. Soc. 67 (1949) 421-432

[6] Ilya Kapovich, Hamish Short, Greenberg's theorem for quasiconvex subgroups of word hyperbolic groups, Canad. J. Math. 48 (1996) 1224-1244

[7] Ilya Kapovich, Daniel T Wise, The equivalence of some residual properties of word-hyperbolic groups, J. Algebra 223 (2000) 562-583

[8] D D Long, Engulfing and subgroup separability for hyperbolic groups, Trans. Amer. Math. Soc. 308 (1988) 849-859

[9] D D Long, G A Niblo, Subgroup separability and 3-manifold groups, Math. Z. 207 (1991) 209-215

[10] D D Long, A W Reid, The fundamental group of the double of the figure-eight knot exterior is GFERF, Bull. London Math. Soc. 33 (2001) 391-396

[11] L M Lopez, Residual finiteness of surface groups via tessellations, Discrete Comput. Geom. 11 (1994) 201-211

[12] Graham A Niblo, Daniel T Wise, The engulfing property for 3 -manifolds, from: "The Epstein birthday schrift", Geom. Topol. Monogr. 1 (1998) 413-418

[13] Peter Scott, Subgroups of surface groups are almost geometric, J. London Math. Soc. (2) 17 (1978) 555-565

[14] Benjamin T Williams, Two topics in geometric group theory, Ph.D. thesis, University of Southampton, England (1998)

[15] Daniel T Wise, The figure-8 knot group is subgroup separable (1998), preprint

Faculty of Mathematical Studies, University of Southampton

Highfield, Southampton SO17 1BJ, UK

Email: G.A.Niblo@maths.soton.ac.uk

Received: 17 May 2002 